S Frisch

Mary Babb Randolph Cancer Center, POB 9300, West Virginia University, Morgantown, WV 26506-9300, USA

* Corresponding author: S Frisch; E-mail: sfrisch@hsc.wvu.edu
1. Screaton RA et al. (2003) Proc. Natl. Acad. Sci. USA 100: 5211-5216

2. Gomez-Angelats M and Cidlowski J (2003) Cell Death Differ. 10: 791-797

3. O'Reilly LA et al. (2004) Cell Death Differ. advance online publication

4. Gurney Jr T and Collard MW (1984) Anal. Biochem. 139: 25-34

5. Gurney Jr T and Foster DN (1977) Methods Cell Biol. 16: 45-68

6. Grooteclaes M et al. (2003) Proc Natl Acad Sci USA 100: 4568-4573

\title{
Nuclear localisation of FADD - rebuttal
}

\author{
Cell Death and Differentiation (2004) 11, 1362-1363. doi:10.1038/sj.cdd.4401513
}

\section{Dear Editor,}

We recently published in your journal a paper describing the subcellular localisation and post-translational modifications of caspase-8 and its adaptor FADD (also called Mort1) in unstimulated cells, and in lymphocytes that had been activated with mitogens or treated with FasL. ${ }^{1}$ In their letter, Frisch et al. question some of the conclusions from our work, specifically our conclusion that FADD is located exclusively in the cytoplasm, since they found significant amounts of FADD in the nucleus, at least in adherent cells.

Firstly, it is noteworthy that most results and conclusions from our publication, ${ }^{1}$ namely mitogenic activation- and FasLinduced post-translational modification and recruitment of caspase- 8 and FADD to distinct clusters at the plasma membrane, have not been challenged. Furthermore, the quality of our novel monoclonal antibodies to mouse caspase8 and FADD has not been challenged by Frisch et al., but was in fact reinforced, since they have used these antibodies in their experiments.

We would now like to address in depth the specific issues raised by Frisch et al.: (1) our method for immunofluorescent staining does not allow efficient detection of nuclear proteins, (2) our method for subcellular fractionation entails the risk of losing proteins from the nucleus and (3) a substantial amount of FADD is localised in the nucleus.

(1) Frisch et al. state that immunofluorescent staining of paraformaldehyde-fixed cells permeabilised with saponin does not allow detection of proteins in the nucleus. We now show that our method readily detects p53 in the nucleus of HeLa cells (Figure 1i). In fact, the staining for p53 was at least as strong as that seen in cells permeabilised with Triton-X100 , the procedure recommended by Frisch et al (compare Figure $1 \mathrm{i}$ with $1 \mathrm{k}$ ). It is noteworthy that permeabilisation with saponin has also been used for detection of Ikaros protein in the nucleus. ${ }^{2}$ We used both methods of cell permeabilisation to detect FADD localisation using our mAb $12 E 7$ (Figures 1a-h). In cells permeabilised with saponin, FADD was found both in the cytoplasm (Figure 1a) and in the nucleus (Figure 1b), with cytoplasmic localisation being considerably more evident. In contrast, and in agreement with the results presented by Frisch et al., when cells were permeabilised with Triton-X100, FADD was found predominantly in the nucleus and only to a lesser extent in the cytoplasm (Figures 1e and f). The reasons for this discrepancy are not clear, but it is possible that epitopes recognised by this antibody are only exposed on nuclear FADD after treatment with certain detergents, perhaps due to association of nuclear FADD with other proteins.

(2) Frisch et al. state that our method for subcellular fractionation may be inadequate because some low molecular weight proteins, such as FADD, may be lost from the nucleus because of diffusion through nuclear pores during washing. We recognise that this is a potential risk, but we note that standard nuclear purification protocols

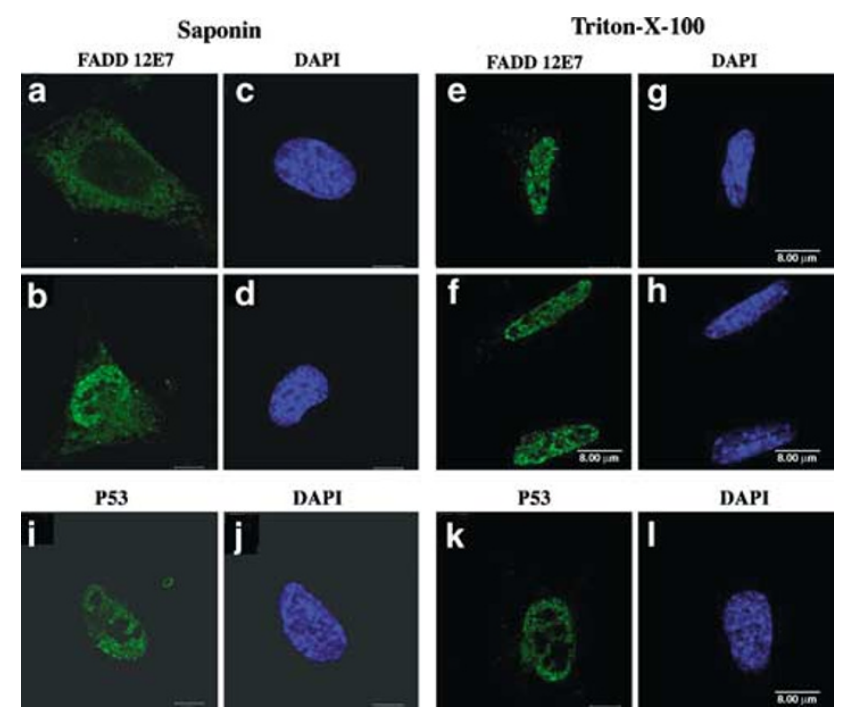

Figure 1 Analysis of FADD subcellular localisation by confocal microscopy. (a, b) FADD (staining with mAb 12E7) is localised in both the cytoplasm and nucleus of HeLa cells permeabilised with $0.3 \%$ saponin. $(\mathbf{g}, \mathbf{h})$ FADD is found in the nucleus and to a lesser extent in the cytoplasm of HeLa cells permeabilised with $0.2 \%$ Triton-X-100. (i, k) HeLa cells stained with anti-P53 mAb (clone 248), showing nuclear localisation after permeabilisation with $0.3 \%$ saponin (i) or $0.2 \%$ Triton-X-100 (k). Cells were stained with anti-FADD mAb 12 E7 (green), detected by biotinylated mouse anti-rat IgG antibodies and FITC-streptavidin (a, b, e, f), or with anti-P53 mAb (248, a gift of Professor D Lane, University of Dundee, Dundee, Scotland) detected with FITC-labelled goat anti-mouse IgG antibodies (i, k) plus DAPI (blue) (c, d, g, h, j, I). Bars represent $8 \mu \mathrm{m}$ 

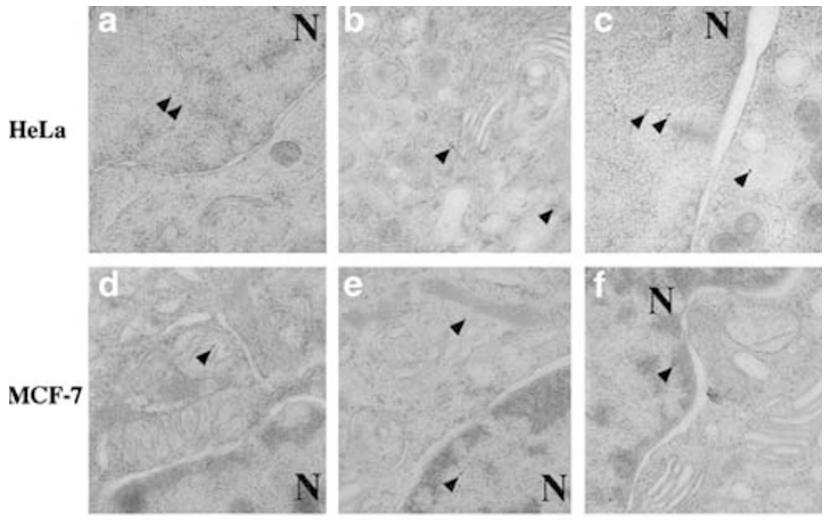

Thy
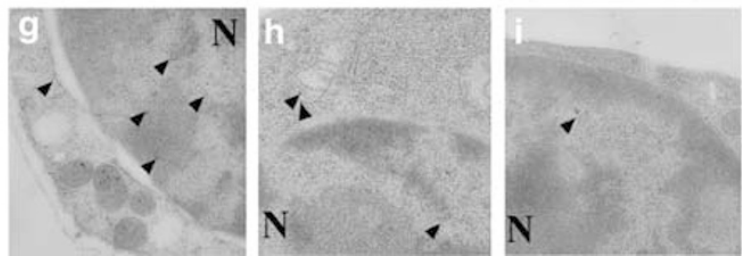

Figure 2 Analysis of FADD subcellular localisation by immunogold electron microscopy. FADD subcellular localisation determined by immunoelectron microscopy in HeLa cells (a-c) and MCF-7 cells (d-f) using rat anti-FADD $\mathrm{mAb} 12 \mathrm{E} 7$ is found in both the nuclear and cytoplasmic compartments. Staining with an Ig isotype-matched control mAb showed no immunogold deposition (data not shown). Materials and Methods: Immunogold electron microscopy. Cells were frozen using a Leica EM high-pressure freezer. Frozen cell pellets were freeze-substituted in $0.1 \%$ uranyl acetate in acetone at $-90^{\circ} \mathrm{C}$ for $48 \mathrm{~h}$ and the temperature raised to $-50^{\circ} \mathrm{C}$ at $6^{\circ} \mathrm{C} / \mathrm{h}$. The cell pellets were rinsed three times in $100 \%$ acetone at $50^{\circ} \mathrm{C}$ for $1 \mathrm{~h}$ each time. Samples were infiltrated with increasing concentrations of HM2O low-temperature resin (ProSciTech, Thuringowa, Australia) in acetone consisting of $25 \%$ ( $8 \mathrm{~h}$ ), $50 \%$ (overnight), $75 \%$ resin $(8 \mathrm{~h}$ ) and $100 \%$ resin (overnight). Infiltrated samples were polymerised in gelatin capsules under UV light for $48 \mathrm{~h}$ at $-50^{\circ} \mathrm{C}$ and brought to room temperature at $6^{\circ} \mathrm{C} / \mathrm{h}$. The sample blocks were further hardened under UV light for $24 \mathrm{~h}$ at room temperature. Embedded cell blocks were sectioned with a diamond knife on a Leica Ultracut R microtome and $90 \mathrm{~nm}$ sections were collected onto pioloformcoated 200 mesh hexagonal gold grids. Prior to immunolabelling, sections were blocked in PBS containing $0.8 \%$ BSA and $0.01 \%$ Tween 80 for $30 \mathrm{~min}$. Sections on grids were then incubated on $20 \mu$ droplets of rat anti-FADD mAbs 7A2 or $12 \mathrm{E} 7$ or rat $\lg \mathrm{G} 1 \kappa$ (isotype control; $\mathrm{BD}$ Pharmingen) $\mathrm{mAb}$, diluted with blocking agent to $10 \mu \mathrm{g} / \mathrm{ml}$ for $4 \mathrm{~h}$ at room temperature. After three washes in blocking agent, grids were incubated overnight at $4^{\circ} \mathrm{C}$ on $20 \mu$ droplets of goat anti-rat IgG antibody (1:40; Jackson ImmunoResearch), conjugated to $18 \mathrm{~nm}$ gold particles. Labelled grids were washed three times, immersed in distilled water and allowed to air dry. Samples were then sequentially stained with saturated uranyl acetate for $15 \mathrm{~min}$ and triple lead for $10 \mathrm{~min}^{6}$ and viewed on a Phillips CM120 Biotwin transmission electron microscope at $120 \mathrm{kV}$

recommend washing of isolated nuclei to minimise contamination with cytoplasmic material. We note that in our original publication we detected, as expected, PARP exclusively in the nuclear fraction and Apaf-1 only in the cytoplasmic fraction of Jurkat and HeLa cells (Figure $4 \mathrm{a}$ and $\mathrm{b}$ in O'Reilly et $a{ }^{1}{ }^{1}$ ), demonstrating the reliability of our protocol for subcellular fractionation.

(3) Owing to the potential for depleting FADD from the nucleus during subcellular fractionation, and owing to the differences in staining for FADD observed in fixed cells permeabilised with either saponin or Triton-X-100, we have now examined FADD localisation in freeze-substituted cell pellets by immunoelectron microscopy. Using this technique, we found FADD in the cytoplasm and the nucleus of adherent HeLa (Figure $2 a-c$ ) and MCF-7 cells (Figure 2d-f) and in nonadherent mouse thymocytes (Figure $2 \mathrm{~g}-\mathrm{i}$ ).

We conclude that FADD is located in the cytoplasm as well as in the nucleus of both adherent and nonadherent cells. The function of FADD in the cytoplasm in apoptosis signalling as an essential component of the death-inducing signalling complex (DISC) of activated 'death receptors', such as Fas/ APO-1/CD95, has been clearly established. ${ }^{3-5}$ It will now be important to define the role of nuclear FADD. Does nuclear FADD translocate to the DISC at the underside of the plasma membrane after 'death receptor' triggering or does it have a role separate from the DISC? Is the role of FADD in the nucleus, akin to its role in the DISC, also related to the activation of caspase-8; that is, is a substantial fraction of caspase- 8 also present in the nucleus? Clarification might be provided by identifying specific binding partners of nuclear FADD or by identifying its nuclear import and export signal sequences. Ultimately, the generation of knock-in mice in which FADD is localised exclusively in the cytoplasm or the nucleus will define the functional roles of FADD in these subcellular compartments and we eagerly await results from such studies.

\section{O'Reilly', S Crawford $^{2}$, DCS Huang ${ }^{1}$ and A Strasser ${ }^{\star, 1}$}

1 The Walter and Eliza Hall Institute of Medical Research, Melbourne, Australia

2 Department of Botany, The University of Melbourne, Melbourne, Australia

* Corresponding author: A Strasser, The Walter and Eliza Hall Institute of

Medical Research, Melbourne, Australia; E-mail: straser@ wehi.edu.au

1. O'Reilly LA et al. (2004) Cell Death Differ. 11: 724-736

2. Cobb BS et al. (2000) Genes Dev. 14: 2146-2160

3. Ashkenazi A and Dixit VM (1998) Science 281: 1305-1308

4. Nagata S (1999) Annu. Rev. Genet. 33: 29-55

5. Strasser A et al. (2000) Annu. Rev. Biochem. 69: 217-245

6. Sato T (1968) J. Electron Microsc. 17: 158-159 